\title{
Norois
}

Environnement, aménagement, société

$231 \mid 2014$

Les modes d'habiter à l'épreuve de la durabilité

\section{Le quotidien peut-il être durable? Routines dans la baie de San Francisco}

Can daily life be sustainable? Thinking about routines from a fieldwork conducted in the San Francisco Bay area

\section{Nathalie Ortar}

\section{(2) OpenEdition}

Journals

\section{Édition électronique}

URL : https://journals.openedition.org/norois/5066

DOI : $10.4000 /$ norois.5066

ISBN : 978-2-7535-3599-2

ISSN : $1760-8546$

Éditeur

Presses universitaires de Rennes

Édition imprimée

Date de publication : 30 septembre 2014

Pagination : 13-25

ISBN : 978-2-7535-3465-0

ISSN : 0029-182X

\section{Référence électronique}

Nathalie Ortar, «Le quotidien peut-il être durable ? Routines dans la baie de San Francisco », Norois [En ligne], 231 | 2014, mis en ligne le 30 juin 2016, consulté le 13 janvier 2022. URL : http:// journals.openedition.org/norois/5066; DOI : https://doi.org/10.4000/norois.5066

(c) Tous droits réservés 


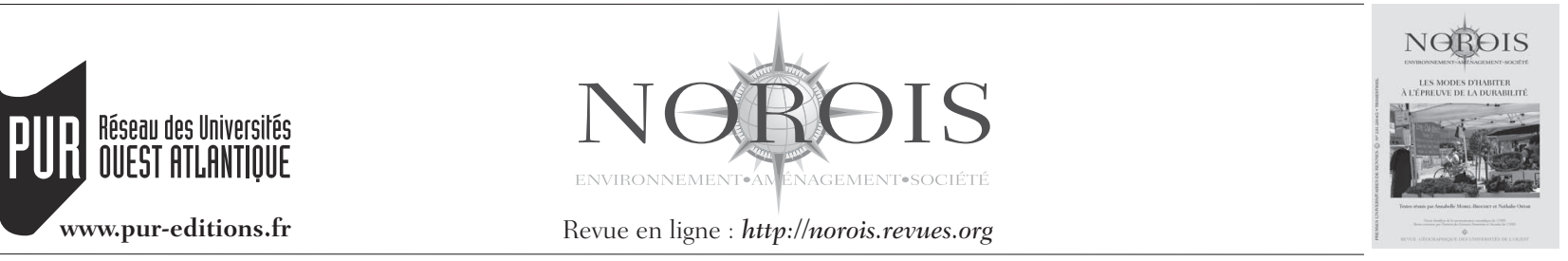

\title{
Le quotidien peut-il être durable? Routines dans la baie de San Francisco
}

\author{
Can Daily Life be Sustainable? \\ Routines in the San Francisco Bay Area
}

\author{
Nathalie Ortar
}

LET, UMR 5593 CNRS, École Nationale des Travaux Publics de l'État,

rue Maurice-Audin - 69580VAuLX-EN-VELIN, France.(nathalie.ortar@entpe.fr)

Résumé : Les pratiques de consommation relèveraient d'un mélange de routinisation et de réflexivité en relation avec des considérations environnementales. Les habitudes s'insèrent de plus dans un ensemble de pratiques affectant le quotidien qui se doivent d'être analysées pour en comprendre le sens. Dans cet article nous souhaitons nous arrêter sur l'entrelacs des routines relevant de différents domaines de la vie de tous les jours, allant de l'achat de nourriture, sa consommation, à la propreté, aux mobilités quotidiennes et de loisirs, aux causes de leur évolution dans le temps, afin de réfléchir au sens des relations existant entre ces domaines ainsi qu'aux différents niveaux de justifications et de silence à partir d'une enquête dans la baie de San Francisco.

\begin{abstract}
Our daily life and ways of inhabiting are shaped by routines. Those routines are both dynamics and immutable, individual and social. To understand the origin of these routines and their mutual articulation, we consider them in the many dimensions of daily life. Understanding how routines change or do not change is a way to understand social evolutions. In this article we will investigate routines - identified or not as being sustainable - and their justification in order to rethink the relations between the various domains of everyday life and their justification. The article is based on a fieldwork conducted in California.
\end{abstract}

Mots clés : habiter - pratiques sociales - développement durable - Californie - méthodologie

Keywords: Inhabiting - social practices - sustainability - California - methodology

Les régimes de transformation des pratiques sociales sont une question récurrente des recherches portant sur le développement durable. Elles ont été documentées en sociologie principalement par les travaux d'Elizabeth Shove (2003) sur les mécanismes à l'origine de nos ressentis et normes, au travers d'une analyse de nos pratiques actuelles mais aussi de l'histoire industrielle et des politiques publiques et commerciales et ceux de Salvador
Juan (2009) sur les trois types d'incohérence écologique en matière de consommation, provenant de la faiblesse de l'offre de produits écologiquement durables, des contradictions personnelles en matière de consommation et d'une interdépendance des usages. Dans son dernier ouvrage, Elizabeth Shove se propose de dépasser ces premières analyses pour accéder à une intelligibilité des pratiques sociales en s'interrogeant sur leur évolution. Il s'agit de 
découvrir les clés de leur création afin de les faire évoluer de nouveau dans une perspective de développement durable (Shove et al., 2012), notamment par une critique de la théorie des choix rationnels. Elizabeth Shove prolonge les travaux théoriques de T. Schatzki (2002) sur l'importance de considérer les pratiques quotidiennes comme relevant d'un nœud d'interconnections entre différents temps et lieux, un apport que nous considérons comme essentiel. Cependant, ces écrits, ceux de T. Schatzki comme ceux d'E. Shove, interrogent à chaque fois une seule dimension du quotidien (la propreté ou le confort par exemple) sans interroger les interactions possibles entre l'ensemble des pratiques ordinaires et routines à l'origine de nos modes d'habiter (MorelBrochet et Ortar, 2012), c'est-à-dire les différents espaces, temps et activités qui fondent et donnent du sens à la vie de tous les jours. En partant de ces constats nous proposons de montrer l'intérêt d'une analyse systémique des pratiques quotidiennes des ménages pour la compréhension des routines et de leurs évolutions à partir d'une recherche menée dans la baie de San Francisco. Cette analyse prend en compte les niveaux macro et micro, qui s'entremêlent au quotidien, l'évolution et la réactualisation du sens donné aux routines, qu'il soit décrit dans les discours ou perçu dans les silences des entretiens. Ces mécanismes permettent d'analyser le manque de consistance en matière de développement durable de certaines des pratiques relevant de différents domaines du quotidien - transport, hygiène, alimentation, etc. - lesquels sont cadrés par des choix - résidentiels, d'alimentation, etc. -, des contraintes professionnelles, des dispositifs marchands et des politiques publiques à l'origine d'injonctions parfois contradictoires.

Pour l'anthropologie du quotidien, « le mot routine vient de route, un petit chemin. Cette métaphore nous fait réfléchir à comment les routines sont créées de la même façon que les chemins, à travers de nombreuses répétitions ", notent B. Ehn et O. Löfgren (2010). Elles relèvent de rituels du quotidien qui facilitent l'action. L'action consiste en le choix intentionnel d'activités dont l'individu est capable d'expliquer la finalité et le "mode de faire », ce qu'Anthony Giddens nomme la conscience discursive (Giddens, 1984). De même, certains choix d'action peuvent créer des habitudes ou routines (Campbell, 1996). La routinisation de l'action est pour Max Weber (2003) une des principales forces du quotidien. La finalité de cette incorporation est notamment de réduire l'incertitude lors de la prise de décision (Ilmonen, 2013) et de structurer le quotidien (Ehn et Löfgren, 2010). L'intégration de tâches, de modes de faire et d'objets au quotidien est le processus de base de la construction de la vie individuelle et sociale. Par la répétition, les nouveautés se transforment en habitudes ne sollicitant plus d'effort particulier et deviennent progressivement non-pensées pour reprendre les termes d'Albert Piette (2009). Les routines agissent alors comme des mémoires du corps. Leur revers est qu'elles deviennent dès lors des limites à l'action (Ilmonen, 2013) car en changer demande un effort. Pour résumer les routines servent à réduire la complexité lors d'une prise de décision, à créer un environnement stable, et en ce sens habitable, et à rendre notre comportement prédictible (Ilmonen, 2013).

Les routines ou habitudes qui dominent le quotidien peuvent être accueillies explicitement par les membres de la maisonnée ou incorporées tacitement au sein des familles et des régimes quotidiens. Elles affectent et sont affectées par une division du travail et des rôles au sein de la maisonnée et font l'objet de discussions entre les membres de la famille. Les routines sont aussi ce qui distingue les modes d'habiter entre maisonnées. Selon M. Douglas (1991), ce qui caractérise une maisonnée n'est pas une construction de quatre murs, mais un ordre interne avec des règles, des habitudes et des rythmes, un maillage de routines, d'agréments silencieux, de réflexes enracinés. Nos modes d'habiter sont donc structurés par ces routines qui acquièrent un niveau de subconscience et guident notre quotidien. Ces routines sont à la fois dynamiques et immuables, personnelles et sociales. Le fait même que les changements affectant la vie domestique, comme l'approvisionnement, la préparation des repas, la relation à l'énergie, s'effectuent sur le long terme, sans réelle conscience de leur occurrence, n'est pas un obstacle au changement même. Comprendre l'origine de ces routines et leur articulation entre les différentes tâches et dimensions du quotidien est un élément décisif pour accéder à une compréhension plus large des freins au changement des habitudes quotidiennes, mais aussi à une compréhension des évolutions en cours. Bente Halkier (2013) suggère que le degré d'autonomie et la conditionnalité des 
habitudes sont dépendants des caractéristiques spécifiques des acteurs et du contexte qui se rapporte directement aux possibilités créées par les relations sociales au quotidien. Pour cet auteur, le quotidien se caractérise par sa contingence aussi la vie sociale n'est-elle jamais entièrement fortuite ou déterminée. Les pratiques de consommation sont un mélange de routinisation et de réflexivité en relation avec des considérations environnementales. Les habitudes s'insèrent de plus dans un ensemble de pratiques affectant le quotidien qui se doivent d'être analysées pour en comprendre le sens. Dans cet article nous souhaitons nous arrêter sur l'entrelacs des routines relevant de différents domaines de la vie de tous les jours, allant de l'achat de nourriture, sa consommation, la propreté, les mobilités quotidiennes et de loisirs, aux causes de leur évolution dans le temps, afin d'interroger le sens des relations existant entre ces domaines et à l'intérêt d'une approche systémique dans l'analyse des changements de comportement en lien avec le développement durable. Pour ce faire nous nous appuierons sur une approche méthodologique questionnant les différents niveaux de justifications et de silence dans les entretiens décrivant le quotidien, lesquels sont mis en perspective au regard du contexte sociétal édictant tant des injonctions à la consommation qu'au développement durable. Après une description du contexte de l'enquête nous nous attacherons dans un premier temps à analyser les logiques sous-jacentes aux routines conduisant à une consommation, puis, dans un deuxième temps, aux silences et contraintes entourant les routines et leur apprentissage dans le contexte de la société californienne.

\section{ConTexte}

Au regard de l'ensemble des États-Unis, la Californie fait figure d'État exemplaire de par sa volonté de répondre aux exigences d'un développement plus soutenable et possède une longue tradition de sensibilité à l'écologie, une attitude qui tranche dans un contexte où, à un niveau fédéral, lors de notre séjour en 2010-2011, une partie des Républicains niait toujours la réalité du changement climatique, militant pour un développement croissant de l'exploitation des sables bitumeux, le maintien des aides financières accordées à l'industrie pétrolière, et luttant contre toute baisse du niveau d'émission de gaz à effet de serre qui pouvait avoir un impact sur le mode de vie. Plus largement, les États-Unis ont utilisé la consommation de l'objet neuf comme moyen ultime d'assurer le plein rendement de leur économie et de dégager des profits destinés à favoriser l'essor d'une classe moyenne. Un modèle économique si largement désiré que les États-Unis se sont auto-proclamés « A consumers' republic »(Cohen, 2003).

La Californie s'est dotée en 2006 d'un outil législatif, le Global Warming Solutions Act (AB32), qui doit lui permettre d'atteindre les prérequis du protocole de Kyoto en matière d'émission de gaz à effet de serre. En 2010, les aires urbaines devaient mettre en place des contrats d'objectifs et des protocoles d'action à compléter d'ici à 2035 portant sur les secteurs de l'économie, du social et de l'environnement. Lors de notre séjour réalisé en tant que Distinguished Visiting Scholar du département d'anthropologie de l'université de San José dans le but d'établir un partenariat de recherche autour du quotidien du développement durable en 2010-2011, l'aire urbaine de San Francisco se caractérisait par son étalement urbain et une faible offre de transports en commun. En ce qui concerne les achats d'électroménager, le système de labellisation «Energy star » livre peu d'informations sur les consommations réelles des produits ${ }^{1}$ et, si les produits étiquetés d'origine biologique sont vendus sur les marchés, ils sont encore peu présents dans les grandes surfaces. Enfin, lors de notre séjour, le développement durable bien que promu par l'État de Californie auprès des métropoles ne faisait pas partie des programmes scolaires, pourtant établis par ce même État sous la direction duquel les manuels scolaires sont édités ${ }^{2}$ et l'écologie n'apparaissait que de façon marginale dans les programmes de sciences de la terre à partir de la $3^{\mathrm{e} 3}$. Un élément révélateur de la faible congruence existante entre les différents secteurs d'action de l'État.

L'enquête s'est déroulée dans la Silicon Valley, une appellation reprise de façon endogène par les

\footnotetext{
1. Un informateur m'expliquera ainsi que pour déterminer les performances réelles des appareils électro-ménagers, il a construit un tableau Excel à partir de la base de données fournie par l'État accessible uniquement par internet. Les données sur la consommation ne sont pas indiquées dans les magasins et rarement fournies par les constructeurs.

2. Dans le système public californien les manuels sont imposés par l'Etat de Californie. Il n'en existe qu'un seul par niveau et par matière à une époque donnée.

3. Entretien réalisé auprès d'un professeur de Sciences et vie de la terre de Santa Clara High School.
} 
habitants résidant sur la rive Est de la baie de SanFrancisco, en 2010-2011. La vallée est un haut lieu de l'innovation technologique. Lors de notre séjour, en raison de la nécessité de la mise en application du Global Warming Solutions Act, la vallée voulait également maintenir une figure de précurseur en matière de développement durable. La vallée était de plus agitée de controverses entourant la création d'une ligne à grande vitesse Los Angeles-San Francisco qui pourrait la traverser à terme. Profitant de ce contexte particulier il nous est apparu pertinent d'interroger les pratiques quotidiennes des habitants de la Silicon Valley et les représentations endogènes liées au développement durable des institutions. Nous ne traiterons pas dans le cadre de cet article de cet aspect de la recherche même si les observations qui ont pu être effectuées lors des conseils municipaux de la ville de Palo Alto et des réunions destinées à mettre en application le Global Warming Solutions Act de la Metropolitan Transportation Commission à San Francisco nourrissent également la réflexion.

L'enquête de terrain effectuée auprès des particuliers a combiné plusieurs méthodes d'investigation. Elle s'appuie sur un corpus de trente-huit entretiens compréhensifs réalisés en deux temps auprès de familles et de célibataires résidant principalement en périurbain, un cadre d'enquête qui s'est imposé en raison de la configuration du peuplement de la vallée, un suivi de groupes désireux de mettre en œuvre une transition énergétique, Transition Palo Alto et Santa Clara Green Action, et une ethnographie du quotidien effectuée par observation participante auprès de cinq familles ayant accepté la présence d'un enquêteur qui s'est accompagné d'un suivi quotidien. L'organisation domestique du quotidien est nécessairement multi située note Sarah Pink (2004), car les interactions au sein du logement ne prennent sens qu'en tenant compte de l'ensemble des lieux qui conditionnent l'approvisionnement et l'organisation de l'unité domestique. Cet élément d'enquête fut complété par l'observation flottante de la vie d'un quartier de Santa Clara, une des agglomérations de la Silicon Valley où nous avons résidé pendant un an. Le premier entretien avait pour objectif de collecter de l'information sur les différentes dimensions du quotidien familial : emploi du temps, transport, propreté, alimentation, suivi d'un tour de la maison. En s'appuyant sur le concept de mode d'habiter (Mathieu, 2012), il s'agissait d'interroger les différentes échelles temporelles et spatiales de l'habiter au travers des pratiques ordinaires ainsi que leurs évolutions. Les personnes enquêtées ont été interrogées sur leur vie quotidienne sans que soit mentionné le développement durable ou des pratiques ad hoc. La discussion portait sur l'ensemble du quotidien : depuis la composition familiale, les caractéristiques socio-professionnelles des membres du ménage, les logements successifs, leurs caractéristiques, les travaux, jusqu'aux routines des maisonnées. Le deuxième entretien, qui avait lieu environ un mois après le premier, était destiné à approfondir la description des routines et leur mode d'acquisition au travers des agendas quotidiens, des voyages et des navettes quotidiennes, de l'approvisionnement et de la préparation culinaire, du nettoyage de la maison et du linge, du choix et de l'usage des appareils électro-ménagers. Si le développement durable n'était jamais évoqué dans le guide d'entretien, la plupart des interviewés le mentionnait pour justifier certaines de leurs pratiques ou se démarquer de cette notion à ce stade de l'enquête. Dans l'ensemble les pratiques évoquées relevaient plus d'une écologie du quotidien que du développement durable, mais c'est ce vocable qu'ils utilisaient.

Nous nous appuyons dans notre analyse sur deux exemples étudiés en profondeur destinés à illustrer l'intrication des routines au quotidien, les arbitrages effectués en fonction : de l'organisation temporelle quotidienne, de prescriptions et de valeurs. Ces deux exemples ont été choisis en raison de la qualité des informations recueillies dans les différents domaines du quotidien, permettant ainsi d'illustrer l'intérêt d'une analyse systémique d'unités de consommation pour la compréhension des routines et de leurs évolutions.

\section{Organiser LE QUOTIDIEN : DES ROUTINES ENTRE CHOIX ET CONTRAINTES}

Brent (éducateur spécialisé) et Léa (kinésithérapeute), la trentaine tous les deux, résident dans une banlieue à proximité des centres industriels High Tech de la Silicon Valley. Lui a eu une éducation nord-américaine, tandis que Léa, issue d'une famille d'immigrés philippins, a reçu une éducation asiatique notamment en ce qui concerne l'alimentation. 
Ils sont plus sensibilisés que la plupart des personnes rencontrées aux questions environnementales. Ils ont choisi leur domicile, une maison de $100 \mathrm{~m}^{2}$, en raison de la proximité des écoles, de leur réputation et de la relative proximité avec leurs emplois, $10 \mathrm{~km}$ pour Léa, 30 pour Brent, ce qui les place parmi les personnes interviewées habitant le plus près de leur lieu de travail. Ils possèdent deux voitures, choisies autant pour leur prix que leurs performances. Ils utilisent le vélo pour les trajets effectués dans le quartier et pour conduire les enfants à l'école, font un compost et cultivent quelques légumes dans leur jardin sans toutefois que la production soit suffisante pour modifier leur consommation. Ils s'approvisionnent en plusieurs lieux, aucun des magasins fréquentés n'étant sur la même zone commerciale. Leurs achats sont principalement réalisés dans un supermarché de proximité où ils achètent pratiquement toute leur épicerie. C'est là que Brent s'arrête le soir pour compléter les courses manquantes que lui a signalé Léa lorsqu'il ne réalise pas des achats de légumes, auquel cas il préfère faire un détour par Whole foods, une chaîne haut de gamme proposant des produits biologiques distant d'une dizaine de kilomètres, où il s'approvisionne également en pain. Brent se rend également une fois par mois dans un magasin Costco proposant des produits en gros à prix discount distant d'une dizaine de kilomètres en dehors de leurs cheminements quotidiens, pour acheter œufs, fromage, beurre et huile d'olive, ce qui leur permet, disent-ils, de réduire leurs emballages. Costco vend également des snacks que Brent affectionne et qu'il ne trouve pas ailleurs. Le choix du magasin relève ainsi autant d'une raison économique assortie d'une justification écologique que de goûts acquis pendant l'enfance pour des produits relevant de la «junk food » désapprouvés par sa conjointe. Lea se rend environ tous les quinze jours chez Trader Joe's une chaîne important des produits de qualité, dont un magasin se situe sur son trajet domicile-travail, pour l'achat de produits surgelés destinés au déjeuner des enfants ${ }^{4}$ ou aux repas prépréparés du soir. Lorsqu'ils arrivent à s'organiser, Brent et Léa se rendent au marché fermier local qui n'a lieu que le jeudi matin pour acheter des fruits et des légumes. L'approvisionnement au quotidien en

4. La plupart des enfants apportent leur déjeuner à l'école et les enfants de Brent et Léa ne font pas exception. Le marché a développé une large gamme de produits pour ces déjeuners nécessairement froids. produits issus pour partie de l'agriculture biologique et si possible de Californie, peu disponibles dans les magasins traditionnels relève d'un véritable travail sollicitant d'acquérir des connaissances sur les types de produits offerts et les lieux où les trouver. Les supermarchés étant rarement côte-à-côte, cela nécessite également une organisation du temps et des déplacements pour y accéder.

Dans leurs achats et leurs pratiques alimentaires, Brent et Léa disent rechercher un équilibre. Le premier équilibre a pour but de transiger entre une exigence d'alimentation issue de l'agriculture biologique, leurs possibilités d'approvisionnement local, leurs contraintes horaires professionnelles, leurs revenus et la dépense énergétique nécessaire pour s'approvisionner. Malgré leur désir d'acheter des produits frais locaux, ils ne sont pas prêts pour autant à parcourir de longues distances pour se les procurer sauf en ce qui concerne quelques produits, comme les baies et les légumes, qu'ils pensent être plus contaminés par les pesticides en raison de leur peau fine. Les bas prix de Costco représentent une économie qui leur permet notamment de s'approvisionner régulièrement au marché ou à Whole foods en produits frais de meilleure qualité mais plus chers que ceux disponibles dans les supermarchés locaux. Enfin les semaines où tous les deux ont des horaires de travail peu compatibles avec leur vie familiale, ils consomment des produits préparés produisant de nombreux emballages et dont ils ne contrôlent pas le contenu et la provenance, deux comportements qu'ils essaient d'éviter le reste du temps. Cette dépense est à leurs yeux compensée par l'attention qu'ils portent le reste du temps à leur consommation alimentaire. Transiger sur la qualité de certains produits et leurs principes permet selon eux de maintenir une liberté de choix sur ceux porteurs du plus grand risque sanitaire, et/ou qu'ils trouvent localement, témoignant de la plasticité de leurs routines et de leur régime axiologique lorsqu'une contrainte supérieure, leur difficulté à gérer le temps par exemple, intervient. La qualité des aliments est ainsi évaluée à l'aune du risque sanitaire et de conséquences écologiques. Leur acquisition procède elle d'arguments relevant de l'économie domestique et d'une gestion du temps où la contingence prime (Lallement, 2010; Lamine, 2008) sur les valeurs (Evans, 2012) et les routines. L'échelonnement des achats est aussi une 
façon d'inclure Brent dans un partage des tâches qui lui demeureraient autrement largement étrangères. En effet, les horaires de travail de Léa sont moins contraignants. Cela lui permet de rentrer plus tôt et de s'occuper des devoirs des enfants et de la supervision du dîner lorsqu'elle n'a pas d'activités, ce qui arrive deux soirs par semaine. Inclure Brent dans les achats lui donne pleinement son rôle d'aidant au quotidien (Castelain-Meunier, 2002) à défaut d'être à l'initiative de la gestion des repas principaux. L'alimentation est ainsi comprise au sein de plusieurs ensembles et systèmes qui agissent sur le mode de consommation (Sluys et al., 1997; Valentine, 1999). On observe ainsi cette difficulté de ce qu'Edwin Zaccaï qualifie de rationalisation des styles de vie (Zaccaï, 2009). En effet, nous relevons un ensemble de convictions qui portent autant sur l'écologie, le bien-être, le sain, issues de différentes éducations qui peuvent entrer en contradiction entre elles. Ces convictions entrent en interaction avec un système économique producteur d'une offre de produits, qui ne correspond pas aux qualités recherchées, mais est disponible localement, ainsi qu'avec des contraintes logistiques liées à des organisations professionnelles, qui interférent avec la sphère domestique, et à une répartition des tâches au sein du couple dont les valeurs ou le niveau d'exigence peut diverger (Valentine, 1999).

\section{Freins au changement}

La maisonnée de Kat (secrétaire, 53 ans) et Dan (technicien, 55 ans), inclut ses enfants adultes Sam, 32 ans, et Liz, 30 ans, Pete le «fiancé » de cette dernière, terme utilisé par Kat, leur bébé, ainsi que Lili, la fille de Sam âgée de six ans, qui vit dans la famille à mi-temps. La concentration des générations au sein d'une même maison est liée aux accidents de la vie des uns et des autres. En effet, au moment de l'entretien, seuls Kat et Dan travaillaient à plein temps. Sam s'est retrouvé au chômage après son divorce, ce qui l'a obligé à retourner vivre chez ses parents. Liz et Pete travaillent à mi-temps et occupent des emplois précaires. Kat et Dan travaillent tous deux à environ $40 \mathrm{~km}$ de là, à San José, où ils ont vécu la plus grande partie de leur vie active. Ils s'en sont éloignés en 1999 pour faire construire. Kat a en charge l'approvisionnement et l'organisation de la production des repas même si c'est Sam qui cuisine la plupart du temps, un arrangement négocié en échange de son hébergement. Kat explique : "J'achète beaucoup d'aliments pré-découpés et prêts à cuire parce que Sam cuisine la plupart des repas. C'est plus facile lorsqu'il a Lili d'acheter des produits déjà à demi-cuisinés ou demi-préparés comme cela, elle peut l'aider. Ainsi, nous achetons des sachets de laitue à la place d'une tête de laitue. Les têtes de laitue ne sont de toute façon pas très nutritionnelles. [...] Elles n'ont pas vraiment de valeur nutritive comparées aux autres salades comme les salades mélangées, les épinards ou la romaine qui sont meilleures pour nous. » Ce passage renvoie à deux dimensions différentes du quotidien : l'art de cuisiner et les connaissances et représentations qui entourent l'alimentation. Pour Luce Giard (1994), cuisiner fait appel à des techniques du corps au sens maussien du terme. Cuisiner fait donc appel à l'esprit, au corps et à la culture. Dans le geste se "superposent invention, tradition et éducation »(Giard, 1994). Ce postulat permet à Isabelle Garabuau-Moussaoui (2002) de conclure que le geste concentre en lui divers niveaux sociaux et particularités individuelles, lesquels sont également selon Luce Giard une caractéristique de la vie sociale. Acheter des aliments déjà prêts à être cuisinés se traduit par une transmission tronquée de la cuisine. La cuisine se transforme en un assemblage d'aliments dont la provenance est plus ou moins maîtrisée. Révélatrice de changements en cours dans les pratiques culinaires quotidiennes, elle se traduit à la fois par un accès élargi à des saveurs non maîtrisées par les cuisiniers au quotidien et par un appauvrissement des pratiques culinaires.

Dans son ouvrage sur Les intermittents $d u$ bio, Claire Lamine remarque que "les propriétés de l'aliment donnent des prises au mangeur comme une falaise donne des prises à celui qui l'escalade, le mangeur a alors prise sur ses choix comme le grimpeur sur sa falaise. L'analyse des situations ordinaires d'achat ou de repas permettent d'identifier les trois dimensions de ces prises : la dimension sensorielle, la dimension cognitive et la dimension axiologique, renvoyant respectivement à l'appréhension par les sens, aux informations et connaissances disponibles et enfin aux valeurs et relations en jeu. Lorsqu'il y a problématisation d'une situation et donc basculement, ces trois dimensions de la prise sont l'objet de rééquilibrages qui permettent de revenir à un rapport non problématique à l'aliment, 
de réduire l'incertitude et de restaurer la confiance » (Lamine, 2008). Pour Kat, l'alimentation est évaluée en fonction de sa valeur nutritive renseignée sur les étiquettes. Soucieuse du fait que Lili et elle-même sont pré-diabétiques, Kat précise : «J'essaye d'acheter uniquement des pains qui contiennent de la farine complète ou de l'avoine ou quelque chose qui n'a pas été raffiné. " Comme elle est attentive à consommer suffisamment de fibres alimentaires, elle achète des marques qui «permettent d'éviter de se poser des questions sur le contenu", ce qui implique qu'elle n'achète que des produits pré-emballés. Kat préfère les marques qui procurent une information nutritionnelle jugée fiable, c'est-à-dire clairement étiquetée. Dans un rapport portant sur l'essor des produits alimentaires produisant une information sur les contenus caloriques, Stephen Martinez (2013) remarque que les marques ont compris le besoin des consommateurs d'être tranquillisés, pas tant sur la qualité que sur le contenu énergétique des produits consommés, dans un contexte de prise de conscience des dangers de l'obésité. La dimension cognitive et axiologique tourne ici autour d'un équilibre protéiné et fibreux : "Si vous voulez quelque chose avec plus de protéines que d'hydrates de carbone, cela vous dira qu'il y a $9 \mathrm{~g}$ de protéines dans la boîte et $36 \mathrm{~g}$ de fibres alimentaires et que cela va baisser votre cholestérol. Cela aura ce genre d'informations sur le devant de la boîte, ainsi c'est plus facile d'identifier la meilleure nourriture, la plus saine. » La nourriture saine devient celle dont il est aisé d'identifier le contenu nutritionnel et non celle qui potentiellement contiendra le moins de produits chimiques. Les deux systèmes d'approvisionnement qui en découlent ne peuvent pas réellement se croiser. Outre le fait que pour accéder à une alimentation biologique il est nécessaire de fréquenter d'autres magasins que ceux où Kat s'approvisionne, les produits issus de l'agriculture biologique sont fréquemment vendus en vrac afin de réduire les emballages. Sur l'étiquetage l'attention est mise sur le détail des ingrédients, leur provenance et l'absence d'OGM dont la mention n'est pas obligatoire. D'une part, Brent et Léa, et d'autre part, Kat illustrent deux systèmes de valeurs où le sain n'est pas porteur du même sens. Cette polysémie d'un même terme rajoute de l'ambiguité et renforce l'intérêt d'analyser le régime axiologique en tenant compte l'ensemble des pratiques. Interrogée sur l'alimentation biolo- gique et la possibilité d'acheter au marché, Kat argue du prix trop élevé de ces produits. À la lumière de ce qu'elle nous dit, ce n'est toutefois pas tant le prix qui pose problème - les produits semi-cuisinés qu'elle achète revenant aussi chers, voire plus chers, que des produits issus de l'agriculture biologique - mais l'absence des référents sur lesquels elle a construit son expertise d'acheteuse et de cuisinière et sur lesquels sont adossées ses routines. Bâtir son alimentation sur des produits issus de l'agriculture biologique impliquerait de changer ses lieux d'approvisionnement et de modifier la pratique culinaire familiale, à commencer par les habitudes culinaires de son fils. Le simple transfert d'une alimentation conventionnelle à une alimentation biologique suppose ainsi la transformation d'un ensemble de routines mais aussi l'acquisition de connaissances sur les aliments, les magasins vendant des produits écologiques, le repérage des produits lui convenant, la recherche de recettes de cuisine adaptées à cette nouvelle alimentation et l'apprentissage ou ré-apprentissage de gestes culinaires de base. Enfin, cela obligerait son fils à se conformer au même processus d'apprentissage. Changer s'avère ainsi coûteux en temps, temps de la formation et du tâtonnement, avant l'acquisition de nouvelles routines, temps supplémentaire, enfin, à accorder à une alimentation dont la contingence, pour reprendre le terme d'Elizabeth Shove (2003), est moindre.

\section{Compartimenter : les silences du quotidien}

Le quotidien est de plus compartimenté ainsi que le note Bente Halkier (2013) et fait l'objet d'une attention sélective selon les domaines. Interrogé sur le lavage du linge, Brent déclare faire attention au nombre de lessives réalisées par semaine. Il utilise systématiquement le sèche-linge mais mentionne cet usage sans le commenter autrement que pour détailler sa façon de procéder : effectuer plusieurs machines à la suite pour ensuite tout plier en une fois. Plusieurs logiques sont à l'œuvre. La première relève de sensations corporelles : lavage et séchage du linge touchent à la purification et à la sensualité (Shove, 2003), la sensation sur le corps du linge séché à l'air ou au sèche-linge n'étant pas la même. Comme l'alimentation, le traitement du linge fait partie d'un système parmi des systèmes 
(Shove, 2003). Modifier le cycle du séchage ou la fréquence du nettoyage du linge de toilette ou de lit a une incidence sur le ressenti au quotidien. Transformer ces pratiques revient dès lors à bousculer aussi l'image de soi (Douglas, 2005). L'usage du sèche-linge conditionne une organisation du temps : Brent groupe en une après-midi le traitement du linge, un temps qu'il devrait organiser différemment s'il mettait le linge à sécher à l'air libre. Le traitement du linge renvoie enfin à une routine acquise jeune et observée pendant l'enfance sur laquelle la réflexivité n’est pas exercée, notamment parce que la consommation des appareils électro-ménagers n'est pas directement observable et estimée.

Le silence dans les entretiens qualitatifs est toujours difficile à interpréter en raison de sa nature même, car il peut relever d'une incompréhension, dont la méconnaissance du sujet peut être l'origine, comme de l'oubli ou du déni volontaire ou entretenu par la société (Poland et Peterson, 1998). Le refus de Brent de s'appesantir sur son usage du sèchelinge relève d'un premier type de silence. Admettre que son usage du sèche-linge consomme, l'obligerait à transformer ses routines quotidiennes et le traitement du linge déborderait alors sur d'autres activités. Brent et Léa ont dû récemment changer leur lavelinge. Ils se sont renseignés sur les performances énergétiques des différents appareils électro-ménagers disponibles sur le marché, pour finir par acheter un appareil peu performant, notamment pour sa consommation d'eau, mais bon marché. L'une des raisons était que le coût de l'eau est inclus dans le forfait calculé sur l'ensemble du lotissement et peut difficilement être impacté, pensent-ils, par leurs propres efforts pour réduire leur consommation. Cette invisibilité des consommations concourt à ne pas les prendre en compte au quotidien (Shove et Chappels, 2013). Leur traitement du linge relève ainsi autant d'une incorporation de routines sur lesquelles la réflexivité n'est pas appliquée que d'une compartimentation (Halkier, 2013) du quotidien entre les pôles de vigilance, ici la nourriture qui affecte directement le corps, et ce sur quoi peu de prise est possible ou qui est rendu invisible. Daniel Miller (2012), dans son essai sur le shopping relève la contradiction qui existe entre ce qu'il qualifie de moral et l'éthique. L'éthique relève du bien-être de la planète ou de personnes étrangères à l'entourage proche, tandis que les questions morales portent sur le bien-être de sa maisonnée. Les questions éthiques portent sur des problèmes abstraits, tandis que les problèmes moraux sont concrets. Aussi, comme les individus sont économes et moraux, leur choix est-il rarement éthique, quel que soit, au final, le prix à payer. L'équilibre recherché au sein du couple comme dans la consommation est composé d’objectifs jugés réalistes destinés à permettre la mise en accord de différents principes : un principe égalitaire au sein du couple, même si dans les faits Léa coordonne l'ensemble, des pratiques dites éco-responsables mises en balance avec le coût écologique (se déplacer pour acheter des produits biologiques ou acheter des produits non biologiques sans se déplacer), financier (lorsqu'il est jugé trop cher de suivre ses idéaux), pratique (lorsque renoncer à un appareil électro-ménager demande à modifier ses habitudes) ou symbolique (lorsque d'éventuelles discussions sur les pratiques risquent de mettre à mal les équilibres atteints). L'attention portée au silence dans les entretiens renseigne sur les contradictions entre les questions morales abordées spontanément, les éventuels conflits d'intérêt observés sont alors explicités, et les questions éthiques passées sous silence dès lors qu'elles entrent en contradiction avec la morale affichée.

\section{Temporalité des apprentissages}

Les routines évoluent toutefois au cours de la vie. Dans le récit de leurs routines, Brent et Léa tout comme Kat font autant appel à des gestes et des habitudes acquis pendant l'enfance, qu'au cours de leur vie adulte. Le sens, voire les valeurs dont elles sont porteuses est susceptible d'évoluer. Brent et Léa mentionnent à de nombreuses reprises leur volonté de faire acquérir à leurs enfants de sept et dix ans des habitudes d'hygiène de vie quotidienne, dont les implications relèvent également d'une attention à l'environnement. En ce qui concerne les repas, Brent et Léa demandent aux enfants de manger équilibré et de finir leur assiette, deux buts complémentaires aux finalités divergentes, l'une portant sur l'hygiène de vie et l'autre sur l'attention aux ressources. Aller à l'école à vélo relève de cette même imbrication d'objectifs, où l'attention à la santé à travers une activité physique régulière, thème particulièrement porté par les programmes d'éducation à la santé Let's move mis en place par Michelle Obama 
en 2010, rencontre le désir d'apprendre aux enfants à se déplacer autrement qu'en voiture, attitude que Brent et Léa qualifient de responsable vis-à-vis de l'environnement.

La mention, lors de l'enquête, des programmes de santé publique promus par Michelle Obama invite à interroger le jeu des multiples acteurs éducatifs entourant l'enfant. Le renforcement de l'acquisition des pratiques par l'instauration d'un programme de normalisation des usages de l'individu participe de ce que Rémi Barbier nomme « la fabrique de l'usager » (2002) par l'État qui s'effectue autant en sensibilisant les publics scolaires que les adultes. Cette mention de la transmission de savoir-faire comme de savoir-être qui s'avère constitutive de notre relation au quotidien peut être prolongée ou non lors de la vie adulte ou encore réinterprétée à la lumière de nouvelles exigences ou besoins de justification.

En effet, l'imbrication des objectifs éducatifs soulevés par Brent et Léa renseigne sur la plasticité des buts assignés et leur possible réinterprétation à la lumière de nouveaux impératifs ou attitudes attendues. Kat parlera longuement de son attention à l'énergie à travers notamment sa chasse aux lumières superflues, une habitude héritée de son enfance. Ces routines, inculquées par ses propres parents, trouvent leur origine dans les réflexes acquis lors des années qui ont suivi la crise économique de 1929, une période de l'histoire qui reste particulièrement vive dans les consciences des informateurs âgés de plus de cinquante ans ${ }^{5}$. $\mathrm{Si}$, avec l'essor de la société de consommation, les modes de vie ne sont plus à l'économie, les réflexes acquis pendant cette crise, puis transmis à la génération suivante perdurent au travers de gestes relatifs à l'usage de l'eau et de l'énergie. Ces gestes acquis pendant l'enfance jusqu'à être incorporés, sont réalisés machinalement au quotidien. La transmission à la génération suivante s'est toutefois avérée difficile dans un contexte d'abondance et de bas prix de l'énergie et des matières premières. Ces comportements reprennent une actualité particulière à la lumière de la sobriété prônée par le développement durable. Ces gestes presque honteux issus du manque trouvent, sous l'impulsion de l'injonction au développement

5. Il s'agit du dernier traumatisme vécu et expérimenté collectivement par l'ensemble des Américains sur le sol Américain, élément qui sera régulièrement mentionné dans les entretiens mais aussi dans le cadre des observations effectuées. durable, un espace de valorisation inédit qui permet à celui qui les applique de pouvoir se valoriser à ses propres yeux et auprès de ses proches, puisque ces gestes sont «good for the planet».

\section{LA SOCIÉTÉ CONTRE LE QUOTIDIEN}

La capacité à réinterpréter les routines tout au long de la vie invite à s’interroger sur les modalités d'acquisition des connaissances sur les pratiques, lesquelles renvoient aux institutions, qui renseignent autant sur les changements que les résistances observées (Halkier, 2013). Kat distingue ainsi les gestes d'économie domestique appris lors de l'enfance, qu'elle peut revaloriser, des connaissances sur le développement durable, essentiellement acquises lors de son emploi de secrétaire dans un bureau d'architectes, puis mises en pratique lors de la construction de sa maison et celles glanées dans les médias, en particulier en ce qui concerne la question d'une alimentation dite équilibrée. À ces trois sources repérées par Kat, nous pouvons ajouter l'État, au travers de programmes ponctuels comme celui mentionné plus haut, ainsi que le marché qui conditionne les habitudes d'achat et prescrit certains choix faute d'alternatives. Ce panorama des instances à l'origine de l'acquisition des routines semble toutefois montrer que si les individus sont exposés à de multiples sources d'informations qui peuvent être contradictoires entre elles, l'apprentissage s'effectue lui principalement pendant l'enfance.

\section{Changement sociétaux et glissement des routines}

Ce constat ne rend toutefois pas compte de la réalité évolutive des pratiques. Nous proposons de nous arrêter sur une des routines qui, dans l'enquête, fait l'objet d'un glissement que nous qualifierons de silencieux. Kat indique ainsi que la famille consomme tous ses dîners à la maison. Toutefois, lorsqu'on lui demande ce qu'elle a mangé au cours des trois derniers jours, les dîners ont été composés d'un repas pris dans un fast-food, d'un autre dans un restaurant chinois et enfin des tacos faits à partir de produits semi-préparés. Kat précise qu'il s'agit d'une pratique inhabituelle chez eux. Toutefois, la plupart des personnes interrogées dans le cadre de l'enquête, quel que soit le jour de la semaine, avaient 
consommé en soirée au moins un dîner à l'extérieur ou un repas à emporter et toutes avaient précisé qu'il s'agissait d'une pratique inhabituelle. Une telle récurrence est la marque de ce que nous pouvons qualifier de changement invisible. Le nombre de repas pris à l'extérieur et présentés comme inhabituels est un indicateur de l'écart entre une norme sociale qui célèbre les repas préparés à la maison et pris ensemble, et une réalité qui se traduit par un accroissement des repas pris ou au moins commandés à l'extérieur que constate les enquêtes du département de l'agriculture américain (Biing-Hwan et Guthrie, 2012).

Interrogée sur ses petits déjeuners, Kat reprend : "Je déteste l'admettre mais pour chaque petit déjeuner j'ai eu un muffin aux noix et à la banane. Nous nous arrêtons à Java express sur la route le matin lorsque nous nous rendons au travail pour acheter un café et un muffin aux noix et à la banane. " Dan et Kat ont commencé à prendre leur petit déjeuner dans leur voiture lorsque le couple a acquis sa maison en 1999. Le temps de trajet plus long qui empiète sur le temps libre, la présence de portes gobelets à l'avant des véhicules et le café drive in à l'entrée de l'autoroute ont contribué à changer leurs habitudes. La voiture est devenue habitée nous dit J. Urry $(1999,2005)$ en raison des multiples technologies qui permettent de se sentir entouré par un environnement domestique. Le cas du petit-déjeuner est particulièrement révélateur des conditions qui, une fois réunies, favorisent les changements de comportements. Elizabeth Shove et al. (2007) soulignent dans The design of everyday life, l'importance des dispositifs socio-techniques, notamment ceux conçus autour de la consommation, qu'il s'agisse du porte-gobelets, des drive in, du dessin de l'opercule des tasses destinées à être transportées ou des aliments cuisinés ou semi-cuisinés, pour transiter vers de nouvelles routines. Le glissement silencieux, car effectué sans réel réflexivité sur le sens du changement, vers ces nouvelles routines acquises sans nécessiter d'apprentissage préalable souligne le rôle de l'environnement, tant physique qu'économique et social, dans l'accompagnement des changements.

\section{Silences sur les impacts environnementaux de la mobilité}

D'autres niveaux de silences existent relevant eux de non-dits sociaux. Lors de la construction de leur maison, Kat travaillait pour un cabinet d'architectes. Ils vivaient alors dans une maison mal isolée. Kat attacha une grande importance à cette question et suivit de près les travaux dont elle parle longuement au cours de l'entretien. Le couple fait attention au rendement de l'électroménager acheté. Le lave-vaisselle flambant neuf consomme peu d'eau et d'énergie. Alors que la famille nettoie parfois à la main, Kat l'évite parce que «le lave-vaisselle est plus efficace ». Les boîtes de conserve sont rincées et recyclées et la famille utilise la poubelle la plus petite procurée par la mairie (32 gallons) $)^{6}$. « Nous recyclons pratiquement tout ce que nous pouvons... Je pense que nous faisons plus que notre part de recyclage et réutilisons tout ce que nous pouvons. Nous sommes informés des zéro émission. Pour une maisonnée, nous essayons vraiment de consommer au minimum. " Ces routines quotidiennes, largement développées au cours de l'entretien, contrastent avec plusieurs silences. Le premier concerne la taille de la maison : plus $200 \mathrm{~m}^{2}$ pour un couple dont les enfants s'apprêtaient à décohabiter ${ }^{7}$. Le deuxième renvoie à l'absence dans le discours du coût écologique des déplacements quotidiens, de plus de $80 \mathrm{~km}$ pour chacun des deux véhicules. Seul le coût financier des trajets est évoqué. Ces deux silences contrastent avec l'abondance des descriptions portant sur l'isolation de la maison engendrant une consommation que Kat juge minime. Une qualité toutefois relative puisqu'elle ne dépasse pas les standards imposés par l'Etat de Californie. Implicitement cette qualité vient compenser la pollution générée par les transports. Le silence sur les navettes est autant un refus de remettre en question une ascension sociale mesurable à la taille de la maison que d'un style de vie basé sur une mobilité effectuée en autosolisme, rendu possible par une variété de politiques publiques en faveur de l'automobile et la suppression des alternatives modales par choix politique ou sous l'action des entreprises du secteur automobile

6. Selon la taille du ménage et ses besoins, il est possible de choisir sa taille de poubelle dans certaines villes.

7. Une taille qui correspond toutefois à la taille moyenne des logements récents selon les services du bureau des recensements [http://www.census. gov/const/C25Ann/sftotalmedavgsqft.pdf]. 
(Dennis et Urry, 2009; Lutz et Lutz-Fernandez, 2010). Lorsque Kat parle d'éviter d'utiliser la voiture, c'est pour reconnaître qu'elle devrait marcher davantage à des fins d'entretien physique. Mais elle argumente immédiatement en défaveur d'une telle solution car, dit-elle, même en fin de semaine, elle manque de temps pour réaliser à pied ses déplacements dans le voisinage alors même que ces trajets ne dépassent pas un quart d'heure de marche à pied. Aussi, lorsque Kat indique ne pas pouvoir prendre le temps de se déplacer à pied, ce n'est pas de temps dont il est question mais d'appétence pour la marche.

\section{Routines et politiques publiques : une relation de dépendance}

L'absence de prise en compte de l'impact des transports sur l'environnement, dans les entretiens et les observations est une donnée récurrente. L'évolution du co-voiturage est à ce titre éclairant. Si le covoiturage augmente ce n'est que depuis 2005 (Chan et Shaheen, 2012) après un long déclin malgré la création depuis le début des années 1970 en Californie de voies prioritaires aux heures de pointes pour les véhicules contenant au moins trois personnes. Les périodes historiques où le covoiturage fit l'objet de politiques publiques de grande envergure eurent lieu pendant la deuxième guerre mondiale afin de limiter la consommation de pneumatiques par les civils, puis en réponse à la crise pétrolière de 1973 à 1974. Les tentatives de mise en place de transport à la demande ont depuis échoué, même si les nouvelles plateformes de transport à la demande utilisant notamment les réseaux sociaux se développent depuis 2004 (Chan et Shaheen, 2012). Si les transports sont une préoccupation majeure pour les autorités publiques de la baie de San Francisco en raison de l'encombrement des voies existantes, mais aussi pour des questions environnementales et de santé publique, ainsi que nous avons pu l'observer lors des réunions de la Metropolitan Transportation Commission, la question n'était pas abordée dans les kiosques municipaux présents sur les marchés de San Jose et San Francisco diffusant de l'information sur le développement durable et les moyens de limiter sa consommation énergétique. L'information sur les modes alternatifs de dépla- cement dépendait de l'activisme d'associations ne produisant de l'information que sur un mode, par exemple le vélo. De même, les événements destinés à promouvoir un changement de comportement dans les transports, tels le Bike to work day, le San Jose ou San Francisco bike party, ont pour instigateurs ces mêmes associations de cyclistes. La couverture médiatique des associations dépend de la motivation des bénévoles et de leur capacité à maîtriser les outils de communication. Dans les écoles la question des conséquences de l'usage de la voiture n'est pas plus abordée. Même s'il est difficile selon Isabelle Garabuau-Moussaoui (2010) d'identifier des relations de causes à effets entre politiques publiques et comportements de consommation, force est de constater que les discours sur le développement durable concernent les champs correspondant à une actualité politique et/ou économique représentant un enjeu peu conflictuel, comme le recyclage, le compostage, les économies possibles d'énergie au sein du foyer, tandis que les politiques entourant le transport, qu'il s'agisse du coût généré par l'entretien des routes ou de la promotion de transports en commun et de voies cyclables, des sujets conflictuels au sein de la société américaine ${ }^{8}$, sont peu présentes sur la scène publique. Ainsi si les transports en commun, routier ou ferré, font l'objet de nombreuses polémiques en raison des coûts d'investissement nécessaires à leur fonctionnement et de leur absence de rentabilité, les subventions entourant le réseau routier et l'essence sont rarement mentionnées sur la scène publique. Ce silence politique et médiatique tend à brouiller les messages alors même que les politiques ont besoin de cohérence et de constance pour aboutir (Owens et Driffil, 2008; Gardner et Stern, 1996) et de facto complique les changements recherchés par ces mêmes politiques.

Le quotidien de Kat et Dan et leurs routines actuelles sont ainsi guidées par la désirabilité sociale attachée à la maison, qui les a conduits à acquérir loin de leur lieu de travail un bien répondant aux standards du moment, et à la faiblesse de l'information sur l'impact et le coût réel des transports. Si Kat compartimente certains domaines de son quo-

8. Cf. Street Fight: The Politics of Mobility in San Francisco de Jason Henderson (2013) pour une description des batailles en cours autour des usages de la route et du redéveloppement des transports en commun aux États-Unis. 
tidien, elle se sert également des domaines les plus vertueux pour compenser moralement les moins vertueux.

\section{Conclusion}

D'un point de vue méthodologique, la prise en compte de la nature des différents silences dans les discours s'appuyant sur une ethnographie minutieuse de la société observée favorise une déconstruction des mécanismes expliquant une « inconsistance » des pratiques entre les différents champs : qu'ils soient cadrés par des dispositifs marchands et/ou des politiques publiques, les discours sur les pratiques permettent d'observer en acte l'appropriation du développement durable qui, « loin de constituer une chaîne causale linéaire, [consiste en] une incorporation reformulée des éléments théoriques, ainsi qu'une renégociation des modes de faire préconisés » (Villalba, 2009), un constat que l'auteur établit pour les institutions mais que nous étendons aux particuliers.

Cette attention portée au contenu et contexte des entretiens renforce l'intérêt d'une analyse systémique des routines des maisonnées qui montre d'une part l'interdépendance du quotidien à des éléments exogènes au ménage, soulignant ainsi, à la suite des travaux de Salvador Juan (2009) et d'Edwin Zaccaï (2009), l'importance d'intégrer les niveaux macro et micro dans l'analyse du quotidien pour comprendre les mécanismes à l'origine des routines et leur dépendance aux valeurs des autres membres de la maisonnée et d'autre part, l'importance de prendre en compte les différents postes de consommation afin d'acquérir une vision globale des changements en cours. Cette analyse souligne le caractère contraignant de l'apprentissage des routines acquises pendant l'enfance, ainsi que leur prégnance et incorporation tout au long de la vie malgré une évolution du caractère nécessaire du geste et du régime axiologique les justifiant. En regard, les routines acquises à l'âge adulte entourant la consommation et les déplacements sont marquées par le caractère impensé des changements grâce aux dispositifs sociaux-techniques (Shove et al., 2007) qui les accompagnent. Une aisance d'acquisition qui souligne à rebours l'importance des efforts à fournir pour acquérir de façon volontaire des habitudes de vie venant radicalement transformer le quotidien.
Le constat d'une différence de fonctionnement entre les registres du quotidien permet de nuancer les propos sur l'immobilisme des pratiques, d'analyser la nature des changements en cours et de mieux appréhender les mécanismes et ressorts du quotidien qui favorisent le changement. Intégrer dans l'analyse les contextes de socialisation et leur évolution au cours du temps permet d'appréhender les significations «publiques » et privées données à ces apprentissages et aux gestes incorporés, qu'ils relèvent de pratiques d'économie ou de consommation. En effet, les routines constitutives du quotidien font appel à des valeurs et des habitudes transmises jeune. La recherche d'une congruence avec le développement durable les transforme uniquement lorsqu'elles n'entrent pas en contradiction avec d'autres connaissances et/ou régimes axiologiques, qu'ils soient portés par un modèle de désirabilité sociale, le reflet d'un manque de congruence des politiques publiques, ou le résultat de prescriptions partiellement comprises faute de connaissances adéquates.

\section{Bibliographie}

Barbier R., 2002. La fabrique de l'usager. Le cas de la collecte sélective des déchets, Flux, n 48-49, p. 35-46.

Binng-Hwan L., Guthrie J., 2012. Nutritional Quality of Food Prepared at Home and Away From Home, 1977-2008, Economic Information Bulletin, $\mathrm{n}^{\circ}$ EIB-105, 24 p.

Campbell C., 1996. The Myth of Social Action, Cambridge, Cambridge University Press, 199 p.

Castelain-Meunier C., 2002. La place des hommes et les métamorphoses de la famille, Paris, PUF, coll. «Sociologie d'aujourd'hui », $192 \mathrm{p}$.

Chan N., Shaheen S., 2012. Ridesharing in North America: Past, Present, and Future, Transport Reviews, $\mathrm{n}^{\circ}$ 32-1, p. 93-112.

Cohen L., 2003. A Consumers'Republic. The Politics of Mass Consumption in Postwar America, New York, Vintage Books, $567 \mathrm{p}$.

Dennis K., Unry J., 2009. After the car, Malden, Polity Press, $212 \mathrm{p}$.

Douglas M., 1991. The Idea of a Home: a Kind of Space, Social Research, vol. 58, n 1, 1991, p. 287-307.

Douglas M., 2005. De la souillure. Essai sur les notions de pollution et tabou, Paris, La Découverte, 205 p.

Ehn B., Löfgren O., 2010. The Secret World of Doing Nothing, Berkeley, University of California Press, 304 p.

Evans D., 2012. Beyond the Throwaway Society: Ordinary Domestic Practice and a Sociological Approach to Household Food Waste, Sociology, n² 27, p. 41-58. 
Garabuau-Moussaoui I., 2002. Pour une anthropologie de l'alimentation, in Garabuau-Moussaoui I., Palomares E., Desjeux D. (dir.), Alimentations contemporaines, Paris, L'Harmattan, p. 55-82.

Garabuau-Moussaoui I., 2010. La consommation, entre pratiques, échanges et politique, Sociologies pratiques, $n^{\circ} 20$, p. 1-7.

Gardner G., Stern P., 1996. Environmental Problems and Human Behavior, Boston, Pearson Custom Publishing, $369 \mathrm{p}$.

Giard L., 1994. Faire la cuisine, in De Certau M., Giard L., Mayol P. (dir.), L'invention du quotidien 2. habiter, cuisiner, Paris, Folio Essais, p. 211-350.

Giddens A., 1984. The Constitution of Society: Outline of the Theory of Structuration, Oxford, Polity Press, 402 p.

Halkier B., 2013. Routinisation or Reflexivity? Consumers and Normative Claims for Environmental Consideration, in Gronow J., Warde A. (dir.), Ordinary Consumption, London, Routledge, p. 25-44.

Ilmonen K., 2013. Sociology, consumption and routine, in Gronow J., Warde A. (dir.), Ordinary Consumption, London, Routledge, p. 9-23.

JuAn S., 2009. Introduction. La relation mode de vie - environnement comme question sociologique, in Dobré M., Juan S. (dir.), Consommer autrement. La réforme écologique des modes de vie, L'Harmattan, p. 5-9.

Lallement E., 2010. Ruptures, abandons, déplacements. Ethnologie des pratiques alternatives de consommation et d'échange, Sociologies pratiques, $n^{\circ} 20$, p. 23-36.

Lamine C., 2008. Les intermittents du bio. Pour une sociologie pragmatique des choix alimentaires émergents, Editions de la maison des sciences de l'Homme, Paris/Versailles, Editions Quae, 341 p.

Lutz L., Lutz-Fernandez A., 2010. Carjacked. The culture of the automobile and its effect on our lives, New York, Palgrave Macmillan, $272 \mathrm{p}$.

Martinez S., 2013. Introduction of New Food Products With Voluntary Health- and Nutrition-Related Claims, 19892010, Economic Information Bulletin, n EIB-108, 54 p.

Mathieu N., 2012. Le mode d'habiter. À l'origine d'un concept, in Morel-Brochet A., Ortar N. (dir.), La Fabrique des modes d'habiter: hommes, lieux et milieux de vie, Paris, L'Harmattan, p. 35-53.

Miller D., 2012. Consumption and its consequences, Cambridge, Polity press, $200 \mathrm{p}$.

Morel-Brochet A., Ortar N., 2012. Introduction, in MorelBrochet A., Ortar N. (dir), La fabrique des modes d'habiter. Homme, lieux et milieux de vie, Paris, L'Harmattan, coll. « Habitat et sociétés », p. 13-33.
Owens S., Driffil L., 2008. How to change attitudes and behaviours in the context of energy, Energy Policy, n 36 , p. 4412-4418.

Piette A., 2009. Anthropologie existentiale, Paris, Editions Pétra, $188 \mathrm{p}$.

PInk S., 2004. Home Truths: Gender, Domestic Objects and Everyday Life, Oxford, Berg, 163 p.

Poland B., Peterson A., 1998. Reading between the lines: interpreting silence in qualitative research, Qualitative inquiry, vol. 4, n² 2, p. 293-312.

Schatzki T., 2002. The Site of the Social. A Philosophical Account of the Constitution of Social Life and Change, University Park, The Pennsylvania State University Press, $320 \mathrm{p}$.

Shove E., 2003. Comfort, Cleanliness and Convenience. The Social Organization of Normality, Oxford, Berg, 224 p.

Shove E., Chappells H., 2013. Ordinary Consumption and Extraordinary Relationships: Utilities and their Users, in Gronow J., Warde A. (dir.), Ordinary Consumption, London, Routledge, p. 45-58.

Shove E., Watson M., Hand M., Ingram J., 2007. The Design of everyday Life, Oxford, Berg, 192 p.

Shove E., Pantzar M., Watson M., 2012. The Dynamics of Social Practice: Everyday Life and How it Changes, London, Sage publications, $208 \mathrm{p}$.

Sluys C., Chaudron M., Zaidman C., 1997. Chérie qu'est-ce qu'on mange ce soir ?, Ethnologie française, vol. 27, $\mathrm{n}^{\circ} 1$, p. 87-95.

Urry J., 1999. Automobility, Car Culture and Weightless Travel, a discussion paper, Lancaster University, [http://www. lancs.ac.uk/fass/sociology/research/publications/papers/urryautomobility.pdf].

Unry J., 2005. Sociologie des mobilités, Paris, Armand Colin, $252 \mathrm{p}$.

Valentine G., 1999. Eating in: home, consumption and identity, The Sociological Review, vol. 47, n³ 3, p. 491-524.

Villalba B., 2009. Introduction. Stratégies asymétriques d'appropriation du développement durable, in Villalba B. (dir.), Appropriations du développement durable. Emergences, diffusions, traductions, Lille, Presses Universitaires du Septentrion, p. 13-33.

Weber M., 2003. Economie et société, tome 1: Les Catégories de la sociologie, Paris, Presses Pocket, 410 p.

ZACCAÏ E., 2009. Contradictions de la consommation durable. Les obstacles à une diminution significative d'impacts écologiques sous l'impulsion des consommateurs in Dobré M., JuAn S. (dir.), Consommer autrement. La réforme écologique des modes de vie, Paris, L'Harmattan, p. 13-28. 